AperTO - Archivio Istituzionale Open Access dell'Università di Torino

\title{
Management of myeloma: an Italian perspective
}

\section{This is the author's manuscript}

Original Citation:

Availability:

This version is available http://hdl.handle.net/2318/100188

since 2021-01-27T16:14:19Z

Published version:

DOI:10.1016/j.clml.2011.03.026

Terms of use:

Open Access

Anyone can freely access the full text of works made available as "Open Access". Works made available under a Creative Commons license can be used according to the terms and conditions of said license. Use of all other works requires consent of the right holder (author or publisher) if not exempted from copyright protection by the applicable law. 


\section{(3) \\ UNIVERSITÀ DEGLI STUDI DI TORINO}

This Accepted Author Manuscript (AAM) is copyrighted and published by Elsevier. It is posted here by agreement between Elsevier and the University of Turin. Changes resulting from the publishing process - such as editing, corrections, structural formatting, and other quality control mechanisms - may not be reflected in this version of the text. The definitive version of the text was subsequently published in [Clin Lymphoma Myeloma Leuk. 2011 Jun;11 Suppl 1:S82-6. doi: 10.1016/j.clml.2011.03.026. Epub 2011 May 12].

You may download, copy and otherwise use the AAM for non-commercial purposes provided that your license is limited by the following restrictions:

(1) You may use this AAM for non-commercial purposes only under the terms of the CC-BY-NC-ND license.

(2) The integrity of the work and identification of the author, copyright owner, and publisher must be preserved in any copy.

(3) You must attribute this AAM in the following format: Creative Commons BY-NC-ND license (http://creativecommons.org/licenses/by-nc-nd/4.0/deed.en),

[http://www.sciencedirect.com/science/article/pii/S2152265011000401?via=ihub] 


\title{
Management of Myeloma: An Italian Perspective
}

\author{
Benedetto Bruno,Francesca Gay, Mario Boccadoro, Antonio Palumbo
}

\begin{abstract}
Multiple myeloma remains a fatal plasma cell malignancy. However, new insights into the disease biology and immunology have identified molecular mechanisms, underling functional interactions between plasma cells and the bone marrow microenvironment that have become molecular targets of so-called "new drugs" such as thalidomide, lenalidomide, and bortezomib. Recently, the combinations of new drugs with melphalan and prednisone in elderly patients, and with autologous stem cell transplantation in induction and/or maintenance schedules in younger patients have significantly prolonged overall survival. Optimal combinations and timing are a matter of debate. Moreover, management of side effects is a key clinical target to improve long-term quality of life. Many randomized phase III studies are currently in progress to address these issues. Whether these new advancements in myeloma treatment will eventually translate into a long chronic phase or a monoclonal gammopathy of undetermined significance-like status for the majority of patients remains, however, still unanswered.
\end{abstract}

\section{Introduction}

Multiple myeloma represents the second most common hematological malignancy worldwide and causes about 11,000 and 19,000 deaths every year in the United States and Europe, respectively. ${ }^{1 \text { and } 2}$ The introduction of "new drugs," such as thalidomide, bortezomib, and lenalidomide has significantly improved overall response rates, progression-free survival, and overall survival. ${ }^{3}$ Relapsed patients rescued with these new drugs had longer survival from disease recurrence as compared with those who were not treated with these new therapies (30.9 versus 14.8 months, $P<.001$ ). Moreover, in the past decade, newly diagnosed patients had a $50 \%$ improvement in overall survival as compared with those diagnosed before December 1996, when thalidomide was first introduced (44.8 versus 29.9 months, $P<.001$ ). ${ }^{3}$ Allografting has been regarded as the only potentially curative treatment. ${ }^{4,5}$ and 6 However, the high transplant-related mortality greatly limited its use. ${ }^{7 \text { and } 8}$ Reduced-intensity conditionings, where graft versus myeloma effects play a more important role than the intensity of the preparative regimen, have been explored. ${ }^{9,10,11 \text { and } 12}$ However, results from different groups are conflicting and allografting has become a less attractive option. ${ }^{13,14,15 \text { and } 16}$ Here we present a brief description of the three agents, thalidomide, lenalidomide and bortezomib, that dramatically changed the treatment paradigm of multiple myeloma treatment and focus on a sequential treatment strategy that may translate into high complete remission rates and prolonged overall survival.

\section{"New Drugs" and Their Mechanisms of Action}

\section{Thalidomide and Immunomodulatory Drugs}

Initially, the anti-angiogenic characteristics of thalidomide and the correlation between bone marrow angiogenesis and disease activity formed the empirical basis for its clinical use of in refractory/relapsed myeloma. However, the evidence that bone marrow microvessel density were not significantly changed in responsive patients soon indicated that this drug is also endowed with other mechanisms of action. Thalidomide induces $\mathrm{G}_{1}$ growth arrest and apoptosis in myeloma cells and shows immune-modulatory effects by inducing CD3+ T-cell proliferation, secretion of interferon gamma (IFN- $\gamma$ ) and interleukin 2 (IL-2), and natural killer cell expansion that could 
trigger myeloma cell lysis. ${ }^{17}$ Importantly, thalidomide was soon shown to act in synergy with dexamethasone. ${ }^{18}$ The very first evidence of the clinical efficacy of thalidomide was in patients with heavily pretreated multiple myeloma (MM) refractory to conventional or high-dose chemotherapy. Singhal et al ${ }^{19}$ reported $\geq 25 \%$ reductions in serum or urine paraprotein levels in $32 \%$ of 84 patients. At the time of publication, side-effects included constipation, peripheral neuropathy, weakness, and morning somnolence whereas severe neutropenia was a rare event. This pioneering experience was later updated on a large series of patients and confirmed the encouraging data. ${ }^{20}$

Thalidomide derivatives were later developed. Two major classes of chemical and functional analogues were developed and defined as selective cytokine inhibitory drugs and immunomodulatory drugs. ${ }^{21}$ Among these latter, CC-5013, or lenalidomide, was shown to be up to 2000 times more potent in inducing T-cell proliferation and up to 100 times in enhancing IL-2 and IFN- $\gamma$ secretion. ${ }^{22}$ Richardson et al initially reported a phase I study on 27 patients with relapsed or refractory myeloma. Lenalidomide was given at four daily doses: $5 \mathrm{mg}, 10 \mathrm{mg}, 25 \mathrm{mg}$, and $50 \mathrm{mg}$. ${ }^{22}$ Grade 3 myelosuppression was seen in all patients treated with $50 \mathrm{mg}$, and $25 \mathrm{mg}$ was then considered the maximal tolerated dose. A $\geq 25 \%$ paraprotein reduction was observed in 17 of 24 patients. Importantly, most of them had received prior therapy with thalidomide.

\section{Proteosome Inhibitors}

Bortezomib is the prototype of proteasome inhibitors. ${ }^{23}$ Its molecular target is the $26 \mathrm{~S}$ proteasome, a cytoplasm multisubunit protein complex which regulates the turnover of several intracellular proteins controlling fundamental cell functions such as cell cycle and apoptosis. Bortezomib shows high affinity and specificity for the catalytic activity of the 26S proteasome, the inhibition of which can block protein degradation. Nuclear factor $\kappa \mathrm{B}(\mathrm{NF}-\kappa \mathrm{B})$ is a transcription factor bound to its inhibitory partner protein $\kappa \mathrm{B}(\mathrm{I} \kappa \mathrm{B})$. Once I $\kappa \mathrm{B}$ is phosphorylated in the cytoplasm is eventually degraded by the $26 \mathrm{~S}$ proteasome complex with the release of $\mathrm{NF}-\kappa \mathrm{B}$, that migrates into the cell nucleus and induces the synthesis of anti-apoptotic proteins. The biological NF- $\kappa \mathrm{B}$ functions are blocked by the inhibition of I $\kappa \mathrm{B}$ degradation within the proteosome complex which sequesters NF$\kappa \mathrm{B}$ in the cytoplasm. ${ }^{24}$ and 25 Orlowski et al initially reported a phase I study on 27 patients with advanced hematological malignancies. ${ }^{26}$ Interestingly, 9 patients with advanced plasma cell disorders showed a response including a complete remission in a myeloma patient. Evidence of significant clinical activity was provided by Richardson et al in a phase II study of 202 heavily pretreated myeloma patients. ${ }^{27}$ Responses were seen in 67 patients, including 7 complete remissions with negative immunofixation.

\section{Toxicity Profiles}

Toxicity of new drugs represents a clinical challenge. Venous thromboembolism soon emerged as the most serious side effect of thalidomide in untreated newly diagnosed myeloma patients. ${ }^{28}$ Most thromboembolic episodes occurred early and distant from central venous catheters suggesting a systemic thalidomide-induced prothrombic state. ${ }^{29}$ However, no baseline prothrombotic laboratory abnormalities could be identified. Prophylaxis is now routinely administered in newly diagnosed patients. ${ }^{30}$ Other side effects, such as peripheral neuropathy, numbness, and paraesthesia appeared to correlate with drug dose and treatment duration and should promptly be recognized before neurological damage becomes irreversible.

Lenalidomide has shown a safer toxicity profile. ${ }^{31}$ Myelosuppression may be a serious side effect requiring drug reduction or discontinuation. Every effort should be made to manage adverse events so that patients can remain on treatment to ensure the greatest treatment efficacy. Prolonged neutropenia can effectively be managed by dose modifications and addition of granulocyte-colonystimulating factor (G-CSF), whereas thromboembolic prophylaxis should be considered for all patients. 
Bortezomib-based regimens put patients at risk of peripheral neuropathy, which may be irreversible in a number of patients. In elderly patients, we reduced the incidence of peripheral neuropathy by about $70 \%$ by modifying the administration schedule of bortezomib from days $1,4,8$, and 11 to days $1,8,15$, and 22 . This may be particularly effective for patients who have pre-existing neuropathy. 32 and 33

\section{“New Drugs" In Young Patients}

Nowadays, the definition of a "young patient" is understood not only as patients who are younger than 60 to 65 years of age, but also as those who are older than 65 years but remain medically fit enough to endure intensive and repetitive treatments. After showing their efficacy in refractory/relapsed patients, ${ }^{34,35}$ and 36 new drugs have extensively been used during the induction phase instead of the once standard vincristine-adriamycin-dexametasone (VAD)-based regimens, with the aim of increasing tumor cytoreduction and response rates before autologous transplantation. Most importantly, it is imperative to explore if the initial benefit of higher response rates will also translate into prolonged post-transplant overall survival. Results have so far been rather conflicting.

Lokhorst et al showed a post-transplant benefit in progression-free survival of the combination of thalidomide-adriamycin-dexametasone versus VAD in those patients who reached a very good partial remission but not in those who reached a complete remission after induction. However, there was no difference in overall survival between the two cohorts. ${ }^{37}$ In contrast, Morgan et al reported a prolonged superior benefit in terms of complete remission post-transplant of a combination of cyclophosphamide-thalidomide-dexametasone over cyclophosphamide-VAD. ${ }^{38}$

Lenalidomide with high-dose dexametasone has been shown to be active in newly diagnosed patients. ${ }^{39}$ Moreover, a recent randomized trial showed that lenalidomide with low-dose rather than high-dose dexametasone was associated with less toxicity and better overall survival. ${ }^{40}$ The proteosome inhibitor bortezomib as single agent or in combination with dexametasone has shown potent activity in newly diagnosed myeloma. Harousseau et al compared bortezomibdexamethasone versus VAD. ${ }^{41}$ Both pre- and post-transplant very good partial response rates were superior with bortezomib-dexamethasone as compared to VAD (38\% versus $15 \%$, and $54 \%$ versus $37 \%$, respectively). However, the difference in progression-free survival did not reach statistical significance (36 versus 30 months, respectively). No overall survival benefit has been reported so far. The major adverse effect was the risk of neurotoxicity early in the disease course. Recent reports, however, show that reducing the dose of bortezomib to once weekly shows similar efficacy with significantly lower risk of neurotoxicity. ${ }^{42}$

Multidrug combinations have also been explored. Bortezomib-thalidomide-dexamethasone resulted in better response rates and progression-free survival compared to thalidomide-dexametasone or bortezomib-dexametasone in randomized trials. ${ }^{43}$ and 44 Similarly, the combination of bortezomiblenalidomide-dexamethasone produce high overall and complete remission rates in newly diagnosed patients. ${ }^{45}$ Overall, three-drug combinations appear to improve response rates and progression-free survival compared to two-drug combinations. However, longer follow-up is needed to define if the addition of a third drug results in prolonged overall survival without affecting quality of life.

\section{"New Drugs" In Eldery and Medically Unfit Patients}

The oral combination melphalan-prednisone has been regarded as the standard of care for both elderly and medically unfit patients not eligible for autologous stem cell transplantation for decades. The introduction of immunomodulatory drugs and proteasome inhibitors has radically changed the treatment paradigm and led to new standards of care. To date, five randomized phase III clinical trials have compared melphalan-prednisone with the combination melphalan-prednisonethalidomide. $^{46,47,48,49,50 \text { and } 51}$ All these studies showed a prolonged time-to-progression with the 
latter combination, although in only two this advantage also translated into an improved overall survival. In another trial, the combination of melphalan-prednisone with bortezomib was associated with an increased time-to-progression and overall survival as compared with melphalan-prednisone. Moreover, recent data showed the superiority of the four-drug combination melphalan-prednisonethalidomide-bortezomib followed by bortezomib-thalidomide maintenance over melphalanprednisone-bortezomib, and of melphalan-prednisone-lenalidomide followed by lenalidomide maintenance over melphalan-prednisone alone. ${ }^{32}$ and 52 Importantly, reports showed a reduced toxicity profile of lenalidomide when associated with low doses of steroids rather than the standard doses. ${ }^{40}$ The availability of different efficacious regimens may provide clinicians with the opportunity to tailor a specific approach for each patient in the light of comorbidities and biologic age. Moreover, regional differences in the choice of a given combination may be seen. The use of melphalan-prednisone in association with new drugs remains a predominantly European approach, whereas the use of lenalidomide with low-dose dexamethasone is more commonly used in North America. ${ }^{40}$ Both approaches appear to lead to similar clinical outcomes.

\section{Sequential Approaches for the Treatment of MM}

The recent development of new agents with potent antimyeloma activity that target not only malignant plasma cells but also the myeloma microenvironment has opened a new era of clinical research. However, it does not currently appear that any combinations of these new biologically based drugs allow physicians to reach a cure. Many trials are currently in progress to define the optimal combinations of new drugs with older agents, with/without autologous transplantation, that may provide long-term disease control and translate into significantly prolonged overall survival. Two main approaches may be investigated: one may be that of using three- or even four-drug combinations including novel potent antimyeloma agents in the upfront setting to maximize tumor reduction and the other that of using newer and older agents in a more sequential schedule with the goal of converting the disease into a chronic phase that prolongs survival and improves quality of life.

Richardson et al recently reported the first prospective evaluation of a combination of lenalidomidebortezomib and dexametasone in previously untreated myeloma patients. In this phase I/II study, the maximum planned doses were first established as $25 \mathrm{mg}$ for lenalidomide, $1.3 \mathrm{mg} / \mathrm{m}^{2}$ for bortezomib, and $20 \mathrm{mg}$ for dexametasone. An impressive overall response rate of $100 \%$, including high complete and very good partial remission rates, was reported. ${ }^{53}$ Furthermore, after a median follow-up of 21 months, estimated 18-month progression-free and overall survival for this novel combination with/without autologous transplantation were $75 \%$ and $97 \%$, respectively.

We recently evaluated the effect of sequential approaches in elderly patients and in those who were not eligible for autologous transplantation. ${ }^{32}$ and 54 We investigated the role of bortezomib as induction before autologous stem cell transplantation, followed by lenalidomide as consolidationmaintenance in newly diagnosed elderly patients. ${ }^{54}$ One hundred two patients, 65 - to 75-years old, were enrolled. Induction consisted of four 21 -day cycles of bortezomib $\left(1.3 \mathrm{mg} / \mathrm{m}^{2}\right.$ days $\left.1,4,8,11\right)$, pegylated-liposomal-doxorubicin $\left(30 \mathrm{mg} / \mathrm{m}^{2}\right.$ day 4$)$, and dexamethasone (40 $\mathrm{mg} / \mathrm{d}$ : cycle 1 , days $1-$ 4, 8-11, 15-18; cycles 2-4, days 1-4). Autologous stem cell transplantation included two procedures after melphalan $100 \mathrm{mg} / \mathrm{m}^{2}$ and G-CSF-mobilized stem-cell rescue. Consolidation included four 28-day cycles of lenalidomide ( $25 \mathrm{mg} / \mathrm{d}$ days $1-21$ every 28 days) with prednisone (50 mg every other day), followed by maintenance with lenalidomide $(10 \mathrm{mg} / \mathrm{d}$ days $1-21)$ until relapse. Primary endpoints were safety and efficacy. In a recent analysis, after induction, $58 \%$ of the patients obtained at least a very good partial response including a complete response rate of $13 \%$. Importantly, immunofixation-negative complete remission rates gradually increased to $38 \%$ after the two autologous stem cell transplants and up to $66 \%$ after consolidation with the combination lenalidomide-prednisone and maintenance with lenalidomide alone. At a median follow-up of 21 months, the 2-year progression-free survival and overall survival were $69 \%$, and $86 \%$, respectively. 
During the induction phase, transplant-related mortality was 3\%. Severe grade 3-4 adverse events were thrombocytopenia, neutropenia, peripheral neuropathy, and pneumonia. During the consolidation-maintenance phase, adverse events included primarily neutropenia, thrombocytopenia, pneumonia, and cutaneous rash. To our knowledge, this has been the first sizeable phase II study conducted in newly diagnosed myeloma patients to evaluate the clinical effectiveness of a sequential treatment approach which included new agents with different mechanisms of actions. Bortezomib was used during induction to cyto-reduce the disease before a tandem autologous transplant; lenalidomide was then used to consolidate-maintain post-transplant response. This suggests that a sequential approach may be effective in gradually increasing response rates. Although the importance of "death of response" is not universally accepted, the achievement of higher complete remission or very good partial remission rates is associated with a strong positive impact on overall survival. ${ }^{35}$

A bortezomib-based regimen was investigated in untreated elderly patients by the Spanish group. ${ }^{42}$ Two hundred sixty patients were first randomly assigned to receive six cycles of bortezomibmelphalan-prednisone or bortezomib-thalidomide-prednisone as induction therapy, and then randomly assigned to maintenance therapy with bortezomib-prednisone or bortezomib-thalidomide. The primary endpoint was response rate after induction and maintenance phases. Complete remission rates were $28 \%$ and $20 \%$ after induction, respectively, whereas the rates were $44 \%$ in the bortezomib-thalidomide group and 39\% in the bortezomib-prednisone group after maintenance therapy.

A phase III study on untreated patients ineligible for autologous transplantation by Palumbo et al compared the efficacy of the four-drug combination of bortezomib-melphalan-prednisonethalidomide followed by maintenance with bortezomib-thalidomide with bortezomib-melphalanprednisone without maintenance. ${ }^{32}$ A total of 511 patients were randomized. The primary endpoint was progression-free survival. The 3 -year estimates of progression-free survival were $56 \%$ in patients receiving the four-drug combination with maintenance and $41 \%$ in those receiving the three-drug combination without maintenance $(P=.008)$. Complete response rates were $38 \%$ and $24 \%(P<.001)$, respectively, whereas the 3 -year overall survivals were comparable, at $89 \%$ and $87 \%(P=.77)$, respectively.

Two additional phase II studies in elderly patients that evaluated the clinical efficacy of melphalan plus prednisone combined with bortezomib or lenalidomide showed gradual disease responses; $30 \%$ of the treated patients achieved maximum monoclonal immunoglobulin reduction after 6 months of therapy. These data support treatment plans which include sequential exposure to different drugs to maximize depth of response. ${ }^{52}$ and 55

The role of maintenance has also been explored in young patients undergoing autografting. Thalidomide alone or in combination with corticosteroids used as maintenance after autologous stem cell transplantation has shown promising results in three randomized studies. ${ }^{56,57 \text { and } 58} \mathrm{In}$ another study, maintenance with thalidomide improved progression-free survival but not overall survival. ${ }^{59}$ Long-term treatment with thalidomide and bortezomib inevitably increases the risk of peripheral neuropathy whereas lenalidomide appears more tolerated without the risk of cumulative toxicity. Finally, one study reported a significant increase in complete response rates with a sequential approach, which included consolidation with bortezomib and/or immunomodulatory drugs, although this increase was primarily seen in patients who reached at least a very good partial remission. Thus, consolidation may play its best role in responsive patients. ${ }^{60}$

In summary, although randomized phase III trials comparing different induction and consolidationmaintenance schemas are needed, in our view, the sequential use of new drugs in both young and elderly patients may represent an efficacious treatment paradigm to obtain high complete remission rates and prolonged response duration that may eventually translate into a significant overall survival advantage.

Moreover, serious clinical challenges may lie ahead. MM has been associated with both solid tumors and hematological malignancies such as acute myeloid leukemia and myelodisplastic 
syndromes. ${ }^{61}$ Recently, preliminary data on phase III trials from the Intergroupe Francophone du Myelome, the Cancer and Leukemia Group B, and the Gruppo Italiano Malattie e Matologiche dell'Adulto Myeloma groups, reported a higher than expected incidence of hematological malignancies in the arms that included lenalidomide.

Results are not conclusive, however, the incidence of secondary tumors should carefully be monitored during the long-term follow-up examinations.

\section{Conclusion}

During the past decade, the introduction of new agents with potent antimyeloma activity has changed the treatment paradigm for myeloma cases. A significant improvement in overall survival has undoubtedly been reported in many trials after the incorporation of new drugs as salvage therapy. However, a further overall survival advantage with the use of these agents with/without autologous transplantation as induction therapy remains to be determined in long-term prospective clinical trials. Moreover, some issues in the long-term toxicity management, including the potential emergence of secondary malignancies, of new agents need to be addressed. Sequential use of new therapies with maintenance may represent a novel approach for patients who have MM, and current evidence is moving clinical practice in this direction. The best clinical benefits of the several ongoing clinical trials should become apparent in the next few years.

\section{References}

1

American Cancer Society

Cancer Facts and Figures 2005

American Cancer Society, Atlanta, GA (2005)

\section{2}

P. Boyle, J. Ferlay

Cancer incidence and mortality in Europe, 2004

Ann Oncol, 16 (2005), pp. 481-488

\section{3}

S.K. Kumar, S.V. Rajkumar, A. Dispenzieri et al.

Improved survival in multiple myeloma and the impact of novel therapies

Blood, 111 (2008), pp. 2516-2520

\section{4}

J. Aschan, B. Lonnqvist, O. Ringden et al.

Graft-versus-myeloma effect

Lancet, 348 (1996), p. 6

5

G. Tricot, D.H. Vesole, S. Jagannath et al.

Graft-versus-myeloma effect: Proof of principle

Blood, 87 (1996), pp. 1196-1198

6

R. Le Blanc, S. Montminy-Métivier, R. Bélanger et al. 
Allogeneic transplantation for multiple myeloma: further evidence for a GVHDassociated graft-versus-myeloma effect

Bone Marrow Transplant, 28 (2001), pp. 841-848

7

G. Gahrton, S. Tura, P. Ljungman et al.

Prognostic factors in allogeneic bone marrow transplantation for multiple myeloma

J Clin Oncol, 13 (1995), pp. 1312-1322

8

W.I. Bensinger, C.D. Buckner, C. Anasetti et al.

Allogeneic marrow transplantation for multiple myeloma: an analysis of risk factors on outcome

Blood, 88 (1996), pp. 2787-2793

9

N. Kroger, R. Schwerdtfeger, M. Kiehl et al.

Autologous stem cell transplantation followed by a dose-reduced allograft induces high complete remission rate in multiple myeloma

Blood, 100 (2002), pp. 755-760

10

D.G. Maloney, A.J. Molina, F. Sahebi et al.

Allografting with nonmyeloablative conditioning following cytoreductive autografts for the treatment of patients with multiple myeloma

Blood, 102 (2003), pp. 3447-3454

11

B. Bruno, M. Rotta, F. Patriarca et al.

Non-myeloablative allografting for newly diagnosed multiple myeloma: the experience of the Gruppo Italiano Trapianti di Midollo

Blood, 113 (2008), pp. 3375-3382

12

M. Rotta, B.E. Storer, F. Sahebi et al.

Long-term outcome of patients with multiple myeloma after autologous hematopoietic cell transplantation and nonmyeloablative allografting

Blood, 113 (2009), pp. 3383-3391

13

B. Bruno, M. Rotta, F. Patriarca et al.

A comparison of allografting with autografting for newly-diagnosed myeloma

N Engl J Med, 356 (2007), pp. 1110-1120

14

P. Moreau, F. Garban, M. Attal et al.

Long-term follow-up results of IFM99-03 and IFM99-04 trials comparing

nonmyeloablative allotransplantation with autologous transplantation in high-risk de

novo multiple myeloma

Blood, 112 (2008), pp. 3914-3915 
L. Rosinol, J.A. Pérez-Simon, A. Sureda et al.

A prospective PETHEMA study of tandem autologous transplantation versus autograft followed by reduced-intensity conditioning allogeneic transplantation in newly diagnosed multiple myeloma

Blood, 112 (2008), pp. 3591-3593

16

H. Lokhorst, H. Einsele, D. Vesole et al. International Myeloma Working Group Consensus Statement Regarding the Current Status of Allogeneic Stem-Cell Transplantation for Multiple Myeloma

J Clin Oncol, 28 (2010), pp. 4521-4530

17

P.A. Haslett, L.G. Corral, M. Albert et al.

Thalidomide costimulates primary human $\mathrm{T}$ lymphocytes, preferentially inducing proliferation, cytokine production, and cytotoxic responses in the CD8+ subset J Exp Med, 187 (1998), pp. 1885-1892

18

T. Hideshima, D. Chauhan, Y. Shima et al.

Thalidomide and its analogs overcome drug resistance of human multiple myeloma cells to conventional therapy

Blood, 96 (2000), pp. 2943-2950

19

S. Singhal, J. Mehta, R. Desikan et al.

Antitumor activity of thalidomide in refractory multiple myeloma

N Engl J Med, 341 (1999), pp. 1565-1571

20

B. Barlogie, R. Desikan, P. Eddlemon et al.

Extended survival in advanced and refractory multiple myeloma after single-agent thalidomide: identification of prognostic factors in a phase 2 study of 169 patients Blood, 98 (2001), pp. 492-494

\section{1}

L.G. Corral, P.A. Haslett, G.W. Muller et al.

Differential cytokine modulation and $\mathrm{T}$ cell activation by two distinct classes of thalidomide analogues that are potent inhibitors of TNF-alpha

J Immunol, 163 (1999), pp. 380-386

22

P.G. Richardson, R.L. Schlossman, E. Weller et al.

Immunomodulatory drug CC-5013 overcomes drug resistance and is well tolerated in patients with relapsed multiple myeloma

Blood, 100 (2002), pp. 3063-3067

23

J. Adams, V. Palombella, E.A. Sausville et al. 
Proteasome Inhibitors: a novel class of potent and effective antitumor agents

Cancer Res, 59 (1999), pp. 2615-2631

24

T. Hideshima, C. Mitsiades, M. Akiyama et al.

Molecular mechanisms mediating antimyeloma activity of proteasome inhibitor PS-341

Blood, 101 (2003), pp. 1530-1534

25

T. Hideshima, D. Chauhan, P. Richardson et al.

NF-kappa B as a therapeutic target in multiple myeloma

J Biol Chem, 277 (2002), pp. 16639-16647

26

R.Z. Orlowski, T.E. Stinchcombe, B.S. Mitchell et al.

Phase I trial of the proteasome inhibitor PS-341 in patients with refractory hematologic malignancies

J Clin Oncol, 20 (2002), pp. 4420-4427

27

P.G. Richardson, B. Barlogie, J. Berenson et al.

A phase 2 study of bortezomib in relapsed, refractory myeloma

N Engl J Med, 348 (2003), pp. 2609-2617

28

K. Osman, R. Comenzo, S.V. Rajkumar

Deep venous thrombosis and thalidomide therapy for multiple myeloma

N Engl J Med, 344 (2001), pp. 1951-1952

29

M. Zangari, E. Anaissie, B. Barlogie et al.

Increased risk of deep-vein thrombosis in patients with multiple myeloma receiving thalidomide and chemotherapy

Blood, 98 (2001), pp. 1614-1615

30

A. Palumbo, S.V. Rajkumar, M.A. Dimopoulos et al.

Prevention of thalidomide- and lenalidomide-associated thrombosis in myeloma

Leukemia, 22 (2008), pp. 414-423

31

M. Offidani, P. Leoni, L. Corvatta et al.

Outcome and toxicity in the modern era of new drugs for multiple myeloma: a reappraisal for comparison with future investigational trials

Clin Lymphoma Myeloma Leuk, 10 (2010), pp. 353-360

32

A. Palumbo, S. Bringhen, D. Rossi et al. 
Bortezomib-melphalan-prednisone-thalidomide followed by maintenance with bortezomib-thalidomide compared with bortezomib-melphalan-prednisone for initial treatment of multiple myeloma: a randomized controlled trial

J Clin Oncol, 28 (2010), pp. 5101-5109

33

S. Bringhen, A. Larocca, D. Rossi et al.

Efficacy and safety of once weekly bortezomib in multiple myeloma patients

Blood (2010), pp. 4745-4753

34

P.G. Richardson, P. Sonneveld, M.W. Schuster et al.

Bortezomib or high-dose dexametasone for relapsed multiple myeloma

N Engl J Med, 352 (2005), pp. 2487-2498

35

D.M. Weber, C. Chen, R. Niesvizky et al.

Lenalidomide plus dexamethasone for relapsed multiple myeloma in North America

N Engl J Med, 357 (2007), pp. 2133-2142

36

M. Dimopoulos, A. Spencer, M. Attal et al.

Lenalidomide plus dexamethasone for relapsed or refractory multiple myeloma

N Engl J Med, 357 (2007), pp. 2123-2132

37

H.M. Lokhorst, I. Schmidt-Wolf, P. Sonneveld et al.

Thalidomide in induction treatment increases the very good partial response rate before and after high-dose therapy in previously untreated multiple myeloma

Haematologica, 93 (2008), pp. 124-127

38

G.J. Morgan, D. Faith, O. Roger et al.

Thalidomide combinations improve response rates; results from the MRC IX study

Blood, 110 (suppl; abstr 3593) (2007)

39

S.V. Rajkumar, S.R. Hayman, M.Q. Lacy et al.

Combination therapy with lenalidomide plus dexamethasone (Rev/Dex) for newly

diagnosed myeloma

Blood, 106 (2005), pp. 4050-4053

40

S.V. Rajkumar, S. Jacobus, N.S. Callander et al.

Lenalidomide plus high-dose dexamethasone versus lenalidomide plus low-dose dexamethasone as initial therapy for newly diagnosed multiple myeloma: an open-label randomised controlled trial

Lancet Oncol, 11 (2010), pp. 29-37 


\section{J.L. Harousseau, M. Attal, H. Avet-Loiseau et al.}

Bortezomib plus dexamethasone is superior to vincristine plus doxorubicin plus dexamethasone as induction treatment prior to autologous stem-cell transplantation in newly diagnosed multiple myeloma: results of the IFM 2005-01 phase III Trial J Clin Oncol, 28 (2010), pp. 4621-4629

\section{2}

M.V. Mateos, A. Oriol, J. Martínez-López et al.

Bortezomib, melphalan, and prednisone versus bortezomib, thalidomide, and prednisone as induction therapy followed by maintenance treatment with bortezomib and thalidomide versus bortezomib and prednisone in elderly patients with untreated multiple myeloma: a randomised trial

Lancet Oncol, 11 (2010), pp. 934-941

\section{3}

M. Cavo, P. Tacchetti, F. Patriarca et al.

Bortezomib with thalidomide plus dexamethasone compared with thalidomide plus dexamethasone as induction therapy before, and consolidation therapy after, double autologous stem-cell transplantation in newly diagnosed multiple myeloma: a randomised phase 3 study

Lancet, 376 (2010), pp. 2075-2085

44

P. Moreau, T. Facon, M. Attal et al.

Comparison of reduced-dose bortezomib plus thalidomide plus dexamethasone (vTD) to bortezomib plus dexamethasone (VD) as induction treatment prior to ASCT in de novo multiple myeloma (MM): results of IFM2007-02 study

J Clin Oncol, 28 (suppl; abstr 15) (2010)

45

P.G. Richardson, E. Weller, S. Lonial et al.

Lenalidomide, bortezomib, and dexamethasone combination therapy in patients with newly diagnosed multiple myeloma

Blood, 116 (2010), pp. 679-686

46

A. Waage, P. Gimsing, P. Fayers et al.

Melphalan and prednisone plus thalidomide or placebo in elderly patients with multiple myeloma

Blood, 116 (2010), pp. 1405-1412

47

A. Palumbo, S. Bringhen, T. Caravita et al.

Oral melphalan and prednisone chemotherapy plus thalidomide compared with melphalan and prednisone alone in elderly patients with multiple myeloma: randomised controlled trial

Lancet, 367 (2006), pp. 825-831

48

A. Palumbo, S. Bringhen, A.M. Liberati et al. 
Oral melphalan, prednisone and thalidomide in elderly patients with multiple myeloma: updated results of a randomized controlled trial

Blood, 112 (2008), pp. 3107-3114

49

T. Facon, J.Y. Mary, C. Hulin et al.

Melphalan and prednisone plus thalidomide versus melphalan and prednisone alone or reducedintensity autologous stem cell transplantation in elderly patients with multiple myeloma (IFM 99-06): a randomized trial

Lancet, 370 (2007), pp. 1209-1218

50

C. Hulin, T. Facon, P. Rodon et al.

Efficacy of melphalan and prednisone plus thalidomide in patients older than 75 years with newly diagnosed multiple myeloma: IFM 01/01 trial

J Clin Oncol, 27 (2009), pp. 3664-3670

51

P. Wijermans, M. Schaafsma, F. Termorshuizen et al.

Phase III study of the value of thalidomide added to melphalan plus prednisone in elderly patients with newly diagnosed multiple myeloma: the HOVON 49 Study

J Clin Oncol, 28 (2010), pp. 3160-3166

52

A. Palumbo, P. Falco, P. Corradini et al.

Melphalan, prednisone, and lenalidomide treatment for newly diagnosed myeloma: a report from the GIMEMA - Italian Multiple Myeloma Network

J Clin Oncol, 25 (2007), pp. 4459-4465

53

P.G. Richardson, E. Weller, S. Lonial et al.

Lenalidomide, bortezomib, and dexamethasone combination therapy in patients with newly

diagnosed multiple myeloma

Blood, 116 (2010), pp. 679-686

54

A. Palumbo, F. Gay, P. Falco et al.

Bortezomib as induction before autologous transplantation, followed by lenalidomide as consolidation-maintenance in untreated multiple myeloma patients

J Clin Oncol, 28 (2010), pp. 800-807

55

M.V. Mateos, J.M. Hernández, M.T. Hernández et al.

Bortezomib plus melphalan and prednisone in elderly untreated patients with multiple myeloma: results of a multicenter phase $1 / 2$ study

Blood, 108 (2006), pp. 2165-2172

56

M. Attal, J.L. Harousseau, S. Leyvraz et al.

Maintenance therapy with thalidomide improves survival in patients with multiple myeloma 
Blood, 108 (2006), pp. 3289-3294

57

A. Spencer, H.M. Prince, A. Roberts et al.

Thalidomide improves survival when used following ASCT

Haematologica, 92 (suppl s2; abstr S7b.5) (2007), p. 41

58

H. Ludwig, R. Hajek, E. Tóthová et al.

Thalidomide-dexamethasone compared with melphalan-prednisolone in elderly patients with multiple myeloma

Blood, 113 (2009), pp. 3435-3442

59

G.J. Morgan, G.H. Jackson, F.E. Davies et al.

Maintenance thalidomide may improve progression free but not overall survival; results from the Myeloma IX Maintenance Randomisation

Blood, 112 (suppl; abstr 656) (2008)

60

M. Ladetto, G. Pagliano, S. Ferrero et al.

Major tumor shrinking and persistent molecular remissions after consolidation with bortezomib, thalidomide, and dexamethasone in patients with autografted myeloma

J Clin Oncol, 28 (2010), pp. 2077-2084

61

J. Hasskarl, G. Ihorst, E. De Pasquale et al.

Association of multiple myeloma with different neoplasms: systematic analysis in consecutive patients with myeloma

Leuk Lymphoma, 52 (2011), pp. 247-259

62

M. Attal, V. Lauwers, G. Marit et al.

Maintenance treatment with lenalidomide after transplantation for myeloma: final analysis of the IFM 2005-02

Blood, 116 (suppl; abstr 310) (2010)

63

P.L. McCarthy, K. Owzar, K.C. Anderson et al.

Phase III intergroup study of lenalidomide versus placebo maintenance therapy following single autologous hematopoietic stem cell transplantation (AHSCT) for multiple myeloma: CALGB 100104

Blood, 116 (suppl; abstr 37) (2010)

64

A. Palumbo, M. Delforge, J. Catalano et al. 
A phase 3 study evaluating the efficacy and safety of lenalidomide combined with melphalan and prednisone in patients $>65$ years with newly diagnosed multiple myeloma (NDMM): continuous use of lenalidomide vs fixed-duration regimens Blood, 116 (suppl; abstr 622) (2010) 